\title{
Optimal Discretization based Refinement Criteria for Finite Element Adaption
}

\author{
Steve McFee and Dennis Giannacopoulos \\ Computational Analysis and Design Laboratory \\ Department of Electrical Engineering, McGill University \\ 3480 University Street, Montreal, PQ, H3A 2A7, Canada
}

\begin{abstract}
One of the major research issues in adaptive finite element analysis is the feedback control system used to guide the adaption. Essentially, one needs to resolve which error data to feedback after each iteration, and how to use it to initialize the next adaptive step. Variational aspects of optimal discretizations for scalar Poisson and Helmholtz systems are used to derive new refinement criteria for adaptive finite element solvers. They are shown to be effective and economical for $h-, p$ - and $h p$ - schemes.
\end{abstract}

\section{INTRODUCTION}

Discretization of the problem region is an essential part of all finite element methods. The most efficient distribution of degrees of freedom (DOF) for a problem is that which yields a sufficiently accurate solution for the lowest number of free parameters. Currently, the only practical way to achieve this objective is by using adaptive solution strategies [1-4].

There are three main adaptive schemes: (i) $h$-type, which adapts the size of the elements in the mesh; (ii) $p$-type, which adapts the polynomial order of the elements; and (iii) $h p$-type, which is a hybrid approach that combines the strategies of $h$ and $p$-type. The perennial challenge for all types of adaption in finite element analysis (FEA) has been the efficient use of well-defined optimal solution properties as feedback criteria for guiding the solution process towards accurate results [5]. The purpose of this contribution is to introduce and evaluate new refinement criteria for $h$-, $p$ - and $h p$-adaption, which are based on the variational properties of optimal discretizations for the FEA of scalar Poisson and Helmholtz systems.

\section{OPTIMAL FEEDBA $A_{1} C K$ CRITERIA}

The primary objective of adaptive finite element methods is to compute the solutions to engineering problems to within specified tolerances, at a reasonable cost. To meet this goal, an adaptive method needs to evolve and improve an efficient distribution of DOF over the problem domain. This requires establishing solution error distributions, and then adding DOF to the discretization to correct them. One potential route to successful adaption is to employ local error measures that are closely related to the variational principle used to determine the solution to the finite element problem [6].

The ideal mesh for a given number of DOF, i.e. the mesh that produces the most accurate sclution, will exhibit optimal geometric node locations. For such an ideal discretization, the functional corresponding to the variational formulation of the

Manuscript received July 10, 1995. This work was supported in part by the Natural Sciences and Engineering Research Council of Canada. problem, will not only be stationary with respect to the field solution parameters - but also with respect to variations in the geometric node positions. Therefore, one way to detect and rank regions of inferior discretization in a finite element mesh is to evaluate the sensitivity of the functional to differential displacements of the geometric nodes, as illustrated in Fig. 1.
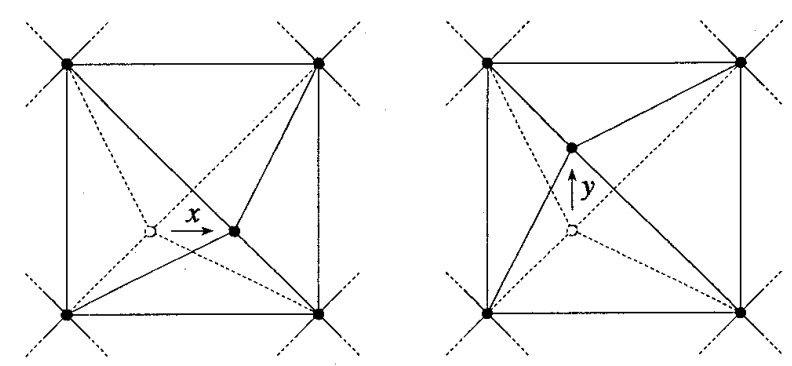

Fig. 1. Differential vertex displacements for $x$ and $y$ directions.

A small perturbation of the position of an element vertex in a region of adequate discretization will result in a relatively small change in the functional value. However, in a region of relatively poor discretization, a small perturbation in vertex positions will yield a significantly larger change. Therefore, by computing the gradients of the functional with respect to vertex positions, it is possible to determine where to improve the discretization, based on a purely local error measure that is closely related the variational principle of the solution.

The gradients of the functional with respect to vertex positions may be computed directly. For Helmholtz systems the $x$-components of the functional gradients may be readily determined from the matrix form:

$$
\frac{1}{2} \boldsymbol{u}^{T} \boldsymbol{V} \boldsymbol{u}-b_{l} \frac{k^{2}}{2} \boldsymbol{u}^{T} \boldsymbol{B} \boldsymbol{u}+b_{i} \boldsymbol{u}^{T} \boldsymbol{B} \boldsymbol{f}
$$

evaluated over the elements connected to the vertex under consideration. Here, $\boldsymbol{u}$ and $\boldsymbol{f}$ are the vectors of the field solution and source term parameters, respectively, and $k$ is the free-space wave number of the system. The square matrix $V$ contains the derivative information that corresponds to the Laplacian part of the functional for vertex $l(l=1,2,3)$ of an element. The entries of $V$ are defined by:

$$
V_{i j}=\frac{1}{4 A^{2}} \sum_{m=1}^{3} \sum_{n=1}^{3}\left[2 A \frac{\partial\left(c_{m} c_{n}\right)}{\partial x_{l}}-\left(b_{m} b_{n}+c_{m} c_{n}\right) b_{l}\right] I_{i j}^{m n}
$$

where $A$ is the element area; $b_{m}$ and $c_{n}$ are the geometric constants related to an element's vertex positions [7]; and $I_{i j}{ }^{m n}$ 
is the integral over the element (in homogeneous coordinates) of the product composed of the derivatives of the $i$ th and $j$ th basis functions, taken with respect to the $m$ th and $n$th simplex coordinates. The gradients of the wave and source terms of the functional are given by the second and third terms in (1), respectively, where $B_{i j}$ is simply the integral over the element (in homogeneous coordinates) of the product of the $i$ th and $j$ th basis functions. The corresponding expressions for the $y$ components of the functional gradients may be obtained by exchanging all the $b$ 's and $c$ 's and by replacing $x_{l}$ with $y_{b}$, in (1) and (2). These Helmholtz results are valid for Poisson systems when the second term in (1) is neglected, and for Laplace systems when the second and third terms are omitted.

It may be confirmed that a wholly equivalent alternative to the explicit computation of the functional gradients for Poisson systems is to evaluate the weighted integral of the scalar potential function $u[8]$ :

$$
\int_{\Omega}\left[\frac{1}{2}|\nabla u|^{2} \nabla g-(\nabla u \cdot \nabla g) \nabla u\right] d \Omega
$$

over the elements connected to the vertex under consideration. The scalar $g$ is continuous and piecewise linear on the mesh: $g=1$ at the vertex in question; $g=0$ at all other vertices. This mathematical equivalence makes it clear that the vertex functional gradients may be interpreted as nodal forces which tend to try to reposition the vertices in a finite element mesh.

Once the gradients of the functional with respect to vertex positions have been computed, they may be used in various ways as error indicators within adaptive solvers. One simple approach is to assess a weighted sum or an average value of the vertex-based functional gradients for each element, then use these values to rank the elements for refinement (Type- $A$ ). A more directed approach is to employ a weighted sum or an average value of the projections of the vertex-based functional gradients onto vectors directed from the vertices towards the centroids of the elements (Type- $B$ ). Unlike the first approach, this scheme depends upon both the directions as well as the magnitudes of the functional gradients. In terms of the nodal force model, this approach uses the net compression forces acting on the elements as the error indicators. In this study, both of these types of methods are investigated to illustrate some of possible ways to exploit the new refinement criteria proposed for adaptive finite element solvers.

\section{RESULTS}

The proposed adaptive refinement criteria were tested and evaluated on two benchmark problems: one Laplace system (Type- $A$ ), and one Helmholtz system (Type- $B$ ). In each case the performance of the scheme was examined with $h-, p$ - and $h p$-adaptive solvers. Finally, the practical significance of the new approach was evaluated using performance comparisons with some of the best adaptive solvers currently available.

The Laplace benchmark system is described by Fig. 2. It is one quarter of a square coaxial line in cross-section - the

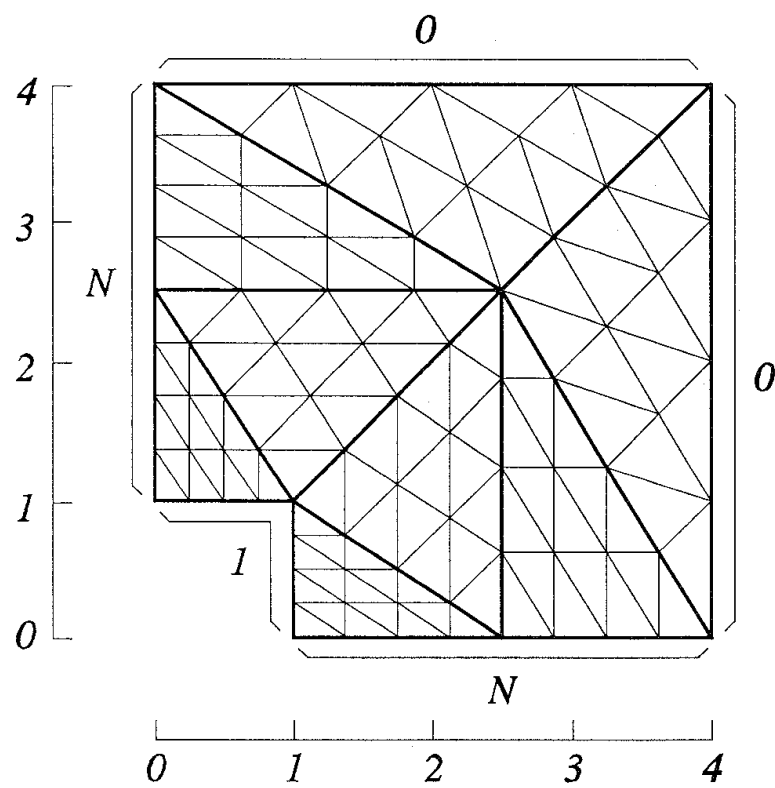

Fig. 2. Laplace; initial $h$-mesh (8 triangles); initial $p$-mesh (128 triangles). standard " $L$ " problem. The conductor boundary conditions are $l$ and $O$ as indicated; and the symmetry planes are labelled $N$. Performance results for the first-order $h$ - and $h p$-adaption studies on functional convergence are presented in Fig. 3. In addition to the uniform $h$-refinement baseline, a practical field discontinuity $h$-adaption result is included for comparison [3]. An example $h$-refined mesh is presented in Fig. 4 to illustrate the sharp focus of the new refinement criteria. The $p$-adaptive performance results are summarized in Table I. In addition to the uniform $p$-refinement baseline, the hierarchal coefficient $p$-adaption result [9] is presented for comparison. The $p$ discretizations ranged from orders 1 through 10 . Finally, an example $p$-refined mesh is illustrated by Fig. 5 , in which the element orders are proportional to the size of the black dots. In this case, the effectiveness of the new refinement criteria for $p$-adaption is shown by the strongly focused and efficient placement of the higher order elements. A 50\% increment in the number of DOF per adaptive step was used to update the

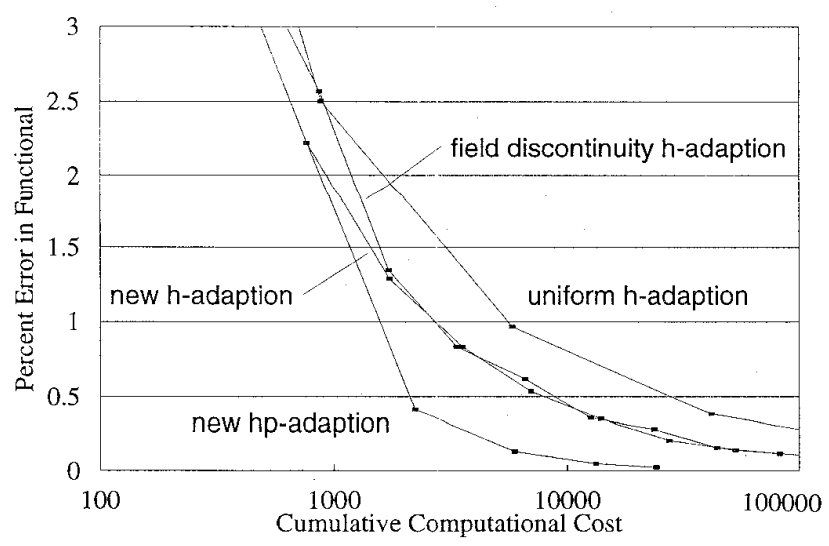

Fig. 3. Cumulative cost of adaption versus percent error in functional. 


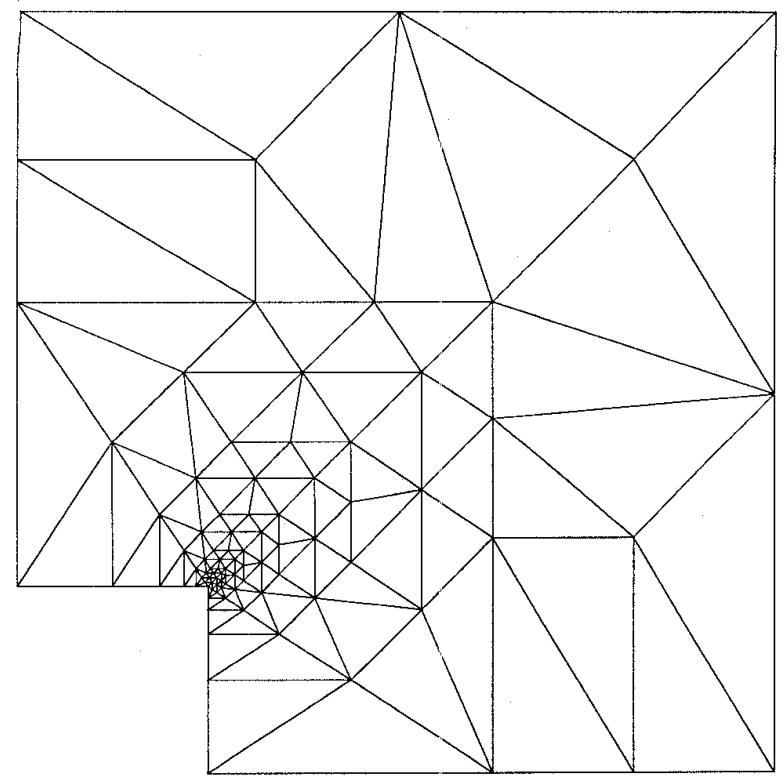

Fig. 4. Example refinement due to new $h$-adaption (second-order run).

TABLE I

Discretization LeVel Versus PERCENT ERror in Functional

\begin{tabular}{ccccc}
\hline Method / \#DOF & $1.00 \%$ & $0.50 \%$ & $0.10 \%$ & $0.05 \%$ \\
\hline uniform p-adaption & 230 & 290 & 1050 & 1670 \\
coef. p-adaption & 230 & 290 & 530 & 675 \\
new p-adaption & 110 & 200 & 550 & 700 \\
new hp-adaption & 100 & 120 & 280 & 375 \\
\hline
\end{tabular}

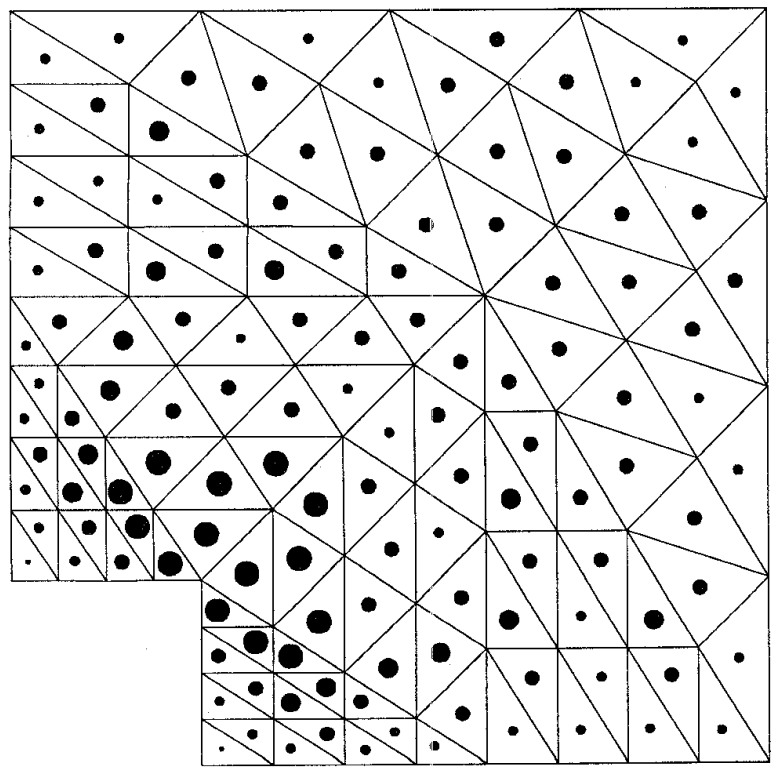

Fig. 5. Example refinement due to new $p$-adaption (orders 1 through 6).

discretizations for the methods considered for this benchmark problem, excluding the uniform refinement procedures.
The Helmholtz benchmark system is described by Fig. 6 . It is an octagonal microstrip patch of size $d$ (34 units), where $\lambda=0.616 d$ ( $\lambda$ is wavelength in the dielectric substrate below the patch). The device has only one port, at the end of the microstrip transmission line connected to the left hand side of the patch. In this study, the boundaries have been modelled as perfect magnetic walls to yield a two-üimensional electric field system. The objective for this benchmark is to find the phase angle of the reflection coefficient at the input port $\boldsymbol{P}$.

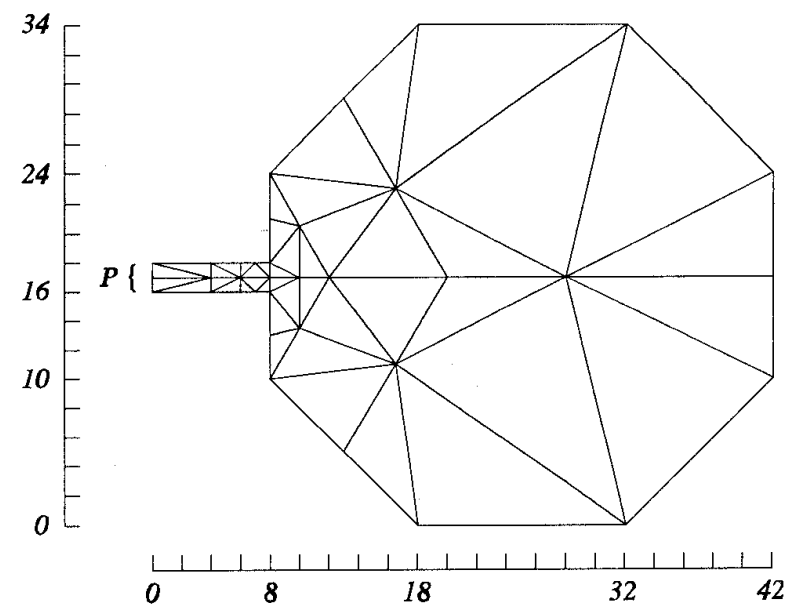

Fig. 6. Helmholtz; initial $h$-mesh and initial $p$-mesh (44 triangles).

The performance results for the $h$ - and $h p$-adaption studies on phase angle convergence are provided in Fig. 7. The uniform $h$-refinement baseline and field discontinuity $h$-adaption results are presented for comparison. For this benchmark, $h$-adaption results for second-order meshes are reported. However, as per the $h p$-adaption in the Laplace study, only the more efficient first-order $h$-followed by $p$-adaption performance is presented for the Helmholtz system. The $p$-adaption results are reported in Table II. The uniform $p$-refinement baseline and hierarchal coefficient $p$-adaption results are presented for comparison.

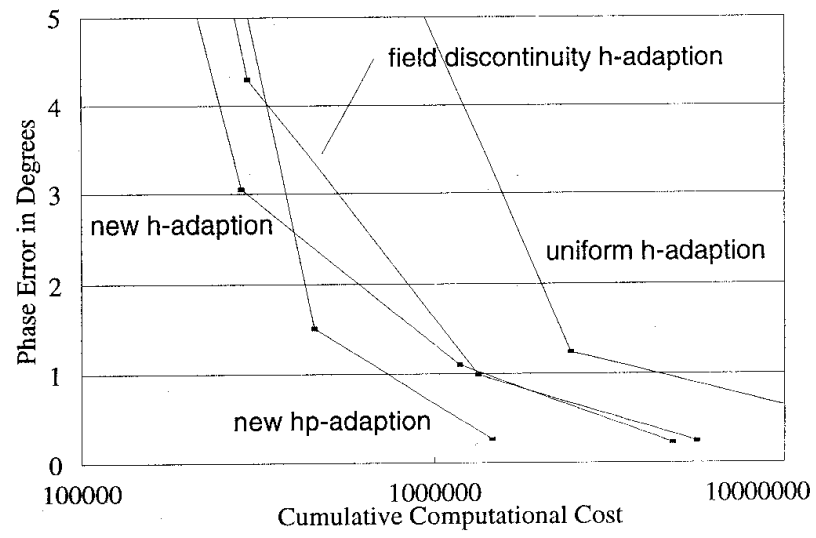

Fig. 7. Cumulative cost of adaption versus reflection phase error.

The $p$-discretizations ranged from orders 1 through 10 . It is interesting to note that the $h p$-adaption is inferior to all the $p$ adaptive methods examined for this benchmark. This result, 
TABLE II

DiscrettZation LeVEL Versus Phase ERror IN DEGREes

\begin{tabular}{ccccc}
\hline Method / \#DOF & $1.5^{\circ}$ & $1.0^{\circ}$ & $0.5^{\circ}$ & $0.1^{\circ}$ \\
\hline uniform p-adaption & 400 & 530 & 690 & 865 \\
coef. p-adaption & 385 & 465 & 550 & 851 \\
new p-adaption & 367 & 405 & 445 & 677 \\
new hp-adaption & 587 & 750 & 920 & 1100 \\
\hline
\end{tabular}

however, is not inconsistent with the undulating nature of the wave solution to the system. A plot of the electric field over the patch is illustrated in Fig. 8. Since the spatial variation of the field is not overly rapid anywhere over the device, and it is reasonably compatible with the DOF distribution provided by the initial $p$-mesh, methods which can efficiently produce a relatively uniform distribution of DOF may be expected to yield the best results. Finally, an example $p$-refined mesh is

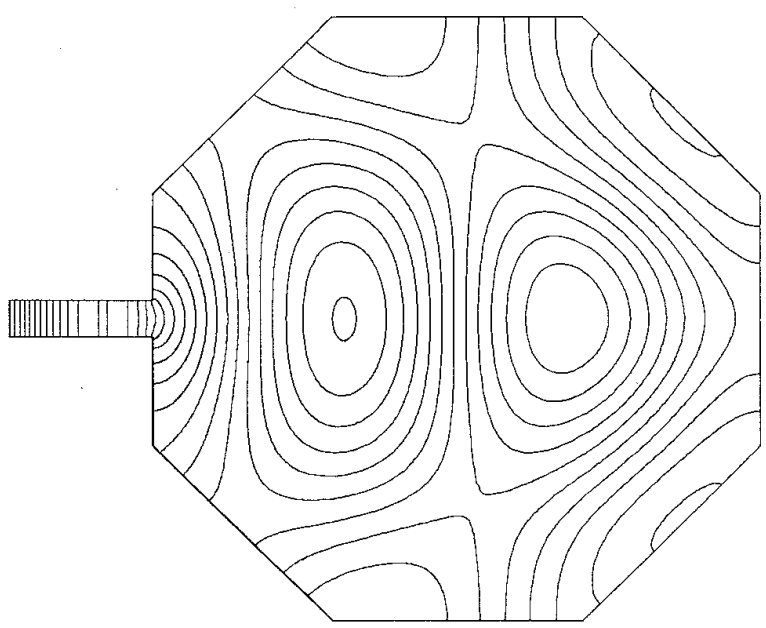

Fig. 8. Converged electric field solution for Helmholtz system.

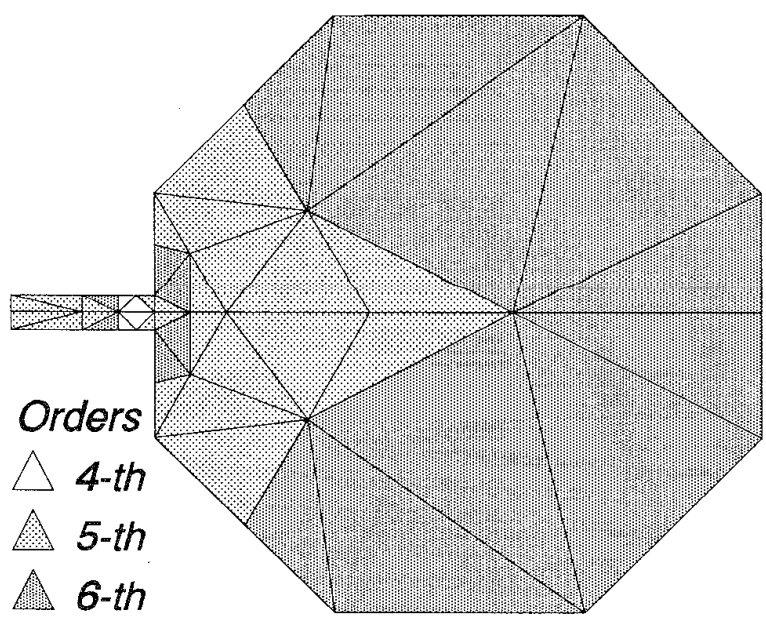

Fig. 9. Example refinement due to new p-adaption. illustrated in Fig. 9. The selectivity of the new refinement criteria, and the way it efficiently distributes the DOF over the problem domain, may be observed by comparing the relative field variations to the relative densities of DOF assigned to the finite element analysis. For this study, a $100 \%$ increase in the number of DOF per adaptive step was used to improve the discretizations for all methods considered, excluding the uniform refinement procedures.

It should be noted that the selected results presented in this section comprise a representative sampling of the full findings obtained over the course of the complete investigation.

\section{CONCLUSIONS}

New refinement criteria, based on variational aspects of optimal discretizations for scalar Poisson and Helmholtz FEA, have been introduced and evaluated for adaption. Stationarity of the functional corresponding to the variational formulation is the fundamental principle essential to the development of the new refinement criteria. Specifically, the gradients of the functional with respect to element vertex positions were used to determine the sensitivity of the functional to differential displacements of the geometric nodes, to distinguish and rank regions of insufficient discretization in a finite element mesh.

The performance results for the benchmark systems that were investigated show that the proposed refinement criteria can be successfully used in adaptive finite element solvers to effectively and economically distribute DOF over the problem domain. In comparison with the state-of-the-art refinement criteria that were evaluated in this research, the new approach produced results that were as good or better, suggesting that further studies involving the new criteria may be warranted.

\section{REFERENCES}

[1] D. Giannacopoulos and S. McFee, "Towards optimal $h-p$ adaption near singularities in finite element electromagnetics", IEEE Trans. Magn., Vol. 30(5), pp. 3523-3526, 1994.

[2] P. Fernandes, P. Girdinio, P. Molfino, G. Molinari and M. Repetto, "A comparison of adaptive strategies for mesh refinement based on a posteriori local error estimation procedures", IEEE Trans. Magn., Vol. 26(2), pp. 795-798, 1990.

[3] J.P. Webb and B. Forghani, "Adaptive improvement of magnetic fields using hierarchal tetrahedral finite elements", IEEE Trans. Magn., Vol. 30(5), pp. 3511-3514, 1994.

[4] K.C. Chellarnuthu and N. Ida, "A Posteriori element by element local error estimation technique and 2D \& 3D adaptive finite element mesh refinement", IEEE Trans. Magn., Vol. 30(5), pp. 3527-3530, 1994.

[5] I. Babuska and R. Rodriguez, "The problem of the selection of an a posteriori error indicator based on smoothening techniques", Int. J. Num. Meth. Eng., Vol. 36, pp. 539-567, 1993.

[6] S. McFee, D.A. Lowther and J.P. Webb, "Forces in finite-element magnetostatics using co-energy related estimation of field accuracy", Jour. Appl. Phys., Vol. 63(8), pp. 3363-3365, 1988.

[7] P.P. Silvester and R.L. Ferrari, Finite Elements for Electrical Engineers, 2nd Edition, Cambridge University Press, Cambridge 1990.

[8] S. McFee, J.P. Webb and D.A. Lowther, "A tunable volume integration method for force calculation in finite-element based computational magnetostatics", IEEE Trans. Magn., Vol. 24(1), pp. 439-442, 1988.

[9] S. McFee and J.P. Webb, "Adaptive finite element analysis of microwave and optical devices using hierarchal triangles", IEEE Trans. Magn., Vol. 28(2), pp. 1708-1711, 1992. 